PAPER

\title{
Cannabinoids in multiple sclerosis (CAMS) study: safety and efficacy data for 12 months follow up
}

\author{
J P Zajicek, H P Sanders, D E Wright, P J Vickery, W M Ingram, S M Reilly, A J Nunn, L J Teare, \\ P J Fox, A J Thompson
}

See Editorial Commentary, p 1612

J Neurol Neurosurg Psychiatry 2005;76:1664-1669. doi: 10.1136/jnnp.2005.070136

See end of article for authors' affiliations .....................

Correspondence to: Professor J P Zajicek Peninsula Medical School, Room N16, ITTC Building, Tamar Science Park, Plymouth, Devon PL6 8BX, UK; john.zajicek@phnt. swest.nhs.uk

Received 25 April 2005 In revised form 15 June 2005 Accepted 2 July 2005
Objective: To test the effectiveness and long term safety of cannabinoids in multiple sclerosis (MS), in a follow up to the main Cannabinoids in Multiple Sclerosis (CAMS) study.

Methods: In total, 630 patients with stable MS with muscle spasticity from 33 UK centres were randomised to receive oral $\Delta^{9}$-tetrahydrocannabinol $\left(\Delta^{9}-\mathrm{THC}\right)$, cannabis extract, or placebo in the main 15 week CAMS study. The primary outcome was change in the Ashworth spasticity scale. Secondary outcomes were the Rivermead Mobility Index, timed 10 metre walk, UK Neurological Disability Score, postal Barthel Index, General Health Questionnaire-30, and a series of nine category rating scales. Following the main study, patients were invited to continue medication, double blinded, for up to 12 months in the follow up study reported here.

Results: Intention to treat analysis of data from the $80 \%$ of patients followed up for 12 months showed evidence of a small treatment effect on muscle spasticity as measured by change in Ashworth score from baseline to 12 months ( $\Delta^{9}-\mathrm{THC}$ mean reduction $1 \cdot 82$ ( $n=154,95 \%$ confidence interval (CI) 0.53 to 3.12 ), cannabis extract $0.10(n=172,95 \% \mathrm{Cl}-0.99$ to 1.19$)$, placebo $-0.23(n=176,95 \% \mathrm{Cl}-1.41$ to 0.94$)$; $p=0.04$ unadjusted for ambulatory status and centre, $p=0.01$ adjusted). There was suggestive evidence for treatment effects of $\Delta^{9}-\mathrm{THC}$ on some aspects of disability. There were no major safety concerns. Overall, patients felt that these drugs were helpful in treating their disease.

Conclusions: These data provide limited evidence for a longer term treatment effect of cannabinoids. A long term placebo controlled study is now needed to establish whether cannabinoids may have a role beyond symptom amelioration in MS.
C annabis and its component cannabinoids (particularly tetrahydrocannabinol $\left(\Delta^{9}\right.$-THC), believed to be the major active cannabinoid) have been claimed anecdotally to improve symptoms related to multiple sclerosis (MS), ${ }^{1}$ although until recently there has been little scientific evidence of effect. There is also experimental evidence that cannabinoids may have a neuroprotective action..$^{2-5}$

The CAMS study ${ }^{6}$ was the first large scale study designed to test the hypothesis that cannabinoids may have a beneficial effect on MS symptoms. The primary outcome measure was the Ashworth assessment of muscle spasticity, and the study also evaluated other MS related symptoms, disability, and safety. The main study covered 15 weeks, with all patients discontinuing treatment during week 14. There was no evidence of treatment effects on change in Ashworth score or other measures of disability from baseline to week 13. However, there was evidence of improvement in walking time for ambulatory patients and in patient perceptions of spasticity, muscle spasms, pain, and sleep. There was evidence of patient unmasking, complicating interpretation of patient assessed outcomes.

Following the main study, patients were given the option of recommencing medication, double blinded, up to week 52. The results from the follow up study are presented here.

\section{METHODS}

The study was approved by the South West Multicentre Research Ethics Committee and conducted under licence from the UK Home Office. All participants provided written informed consent.

\section{Study design}

Details of the main CAMS study have been reported elsewhere. ${ }^{6}$ Patients aged 18-64 years with clinically definite or laboratory supported MS, stable disease for the previous 6 months (in the opinion of the treating physician, rather than as measured by EDSS) and problematic spasticity (Ashworth score of at least 2 in two or more muscle groups), were recruited across 33 UK neurology and rehabilitation centres (see end of paper) between December 2000 and October 2002. Of the group, $97 \%$ had progressive disease and $49 \%$ were ambulant. During the main study, patients attended eight clinic visits over 15 weeks, with a screening and randomisation visit 2-4 weeks before commencement of treatment with either one of two active treatments or their matching placebo, randomly allocated. During the follow up phase (weeks 1652), patients were assessed at three further clinic visits.

\section{Treatment regimens}

Active treatment consisted of either synthetic $\Delta^{9}$-THC (Marinol; Solvay Pharmaceuticals, Atlanta, GA, USA) or a cannabis extract containing $\Delta^{9}$-THC and cannabidiol (CBD) as the main cannabinoids (Cannador; Institute for Clinical Research, IKF, Berlin, Germany). Capsules were manufactured to contain $2.5 \mathrm{mg}$ of $\Delta^{9}$-THC equivalent, $1.25 \mathrm{mg}$ of $\mathrm{CBD}$, and $<5 \%$ other cannabinoids per capsule. Treatment was titrated over the first 5 weeks of the main study, with the

Abbreviations: $\Delta^{9}$-THC, $\Delta^{9}$-tetrahydrocannabinol; $\mathrm{BI}$, Barthel Index; CAMS, Cannabinoids in Multiple Sclerosis; CBD, cannabidiol; GHQ-30, General Health Questionnaire-30; MS, multiple sclerosis; RMI, Rivermead Mobility Index; UKNDS, UK Neurological Disability Score 


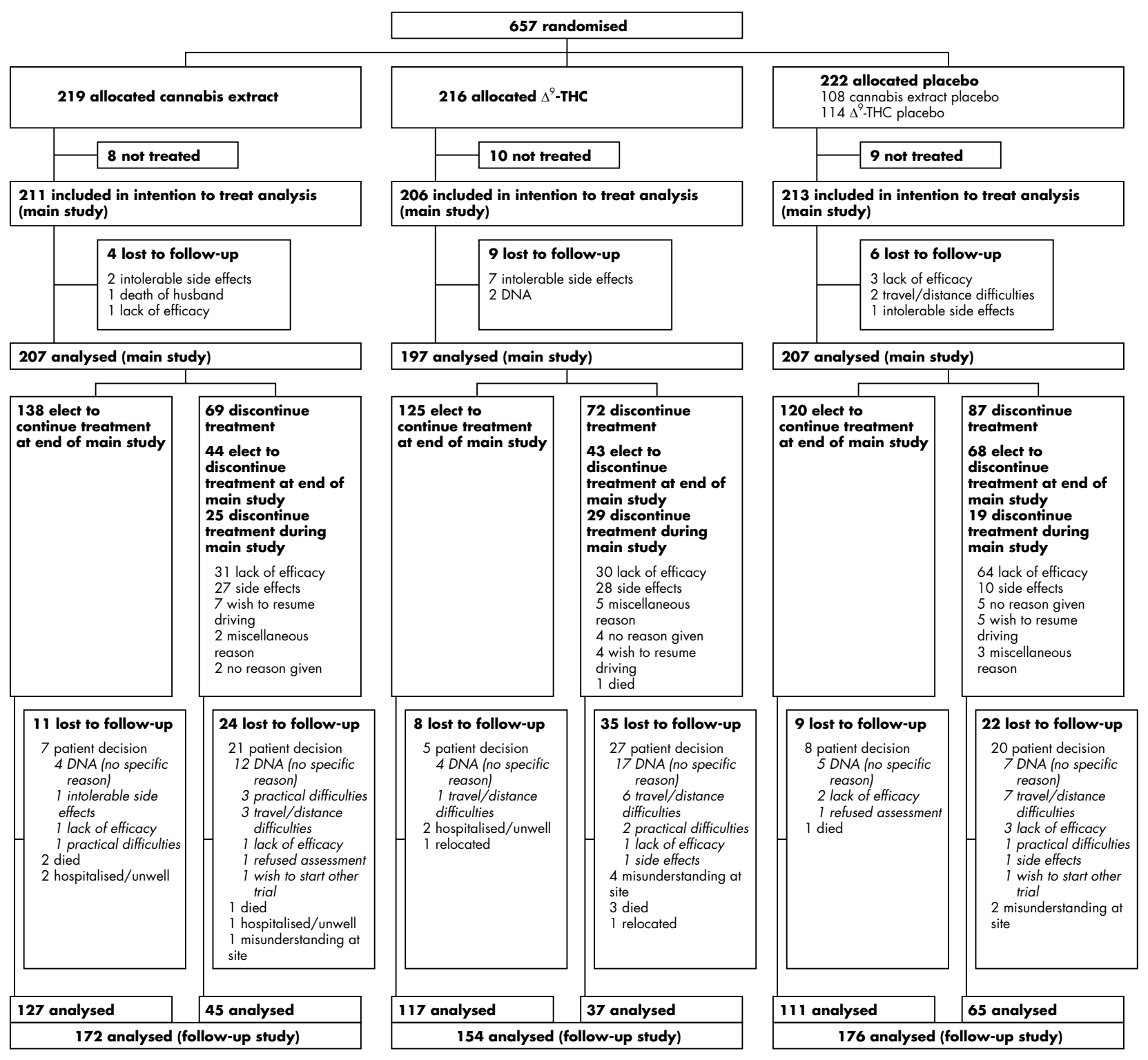

Figure 1 Trial profile.

dose of medication being adjusted, depending on side effects, to a maximum of $25 \mathrm{mg} \Delta^{9}$-THC daily, depending on body weight. Treatment was taken orally in two divided doses. Once optimum dosage had been achieved, patients remained on a steady dose for 8 weeks, before gradually reducing medication to zero during week 14 . Other antispasticity medication was optimised prior to study entry, and any alterations to concomitant medication were documented.

At the end of week 15, patients were offered the opportunity to resume medication at the previously determined dose, for a maximum of 52 weeks. If patients decided not to continue medication, the reasons for non-continuance were documented. Throughout the study, capsule compliance was checked at clinic visits by counting the number of capsules returned. Urine samples for cannabinoid analysis were collected at each clinic visit during the follow up phase in order to identify cannabis use in the placebo group.

\section{Assessments}

In order to reduce the potential for unmasking bias from treatment side effects, two study personnel were used at each centre. A treating physician monitored the dosage, side effects, and patient wellbeing, while an assessor (usually a physiotherapist) measured the Ashworth score, timed 10 metre walk, and Rivermead Mobility Index (RMI). During the follow up phase these data were collected at each of the three monthly visits. Assessors remained blinded to any discussion of dosage or side effects, and when evaluating patients' spasticity, were instructed not to have access to their assessment scores from previous visits. Self completion booklets containing the General Health Questionnaire-30 (GHQ-30), category rating scales (asking patients to assess any change in their symptoms at that point in the study compared with how they were before they started medication, and measuring irritability, depression, tiredness, muscle stiffness, tremor, pain, sleep, muscle spasms, and energy levels), the UK Neurological Disability Scale (UKNDS), and the Barthel Index (BI) were sent to participants just prior to week 27 and week 52. The Expanded Disability Status Scale (EDSS) was used at week 52.

\section{Statistical analysis}

The primary outcome was mean change in Ashworth score from baseline ( start of main study) to end of follow up period 

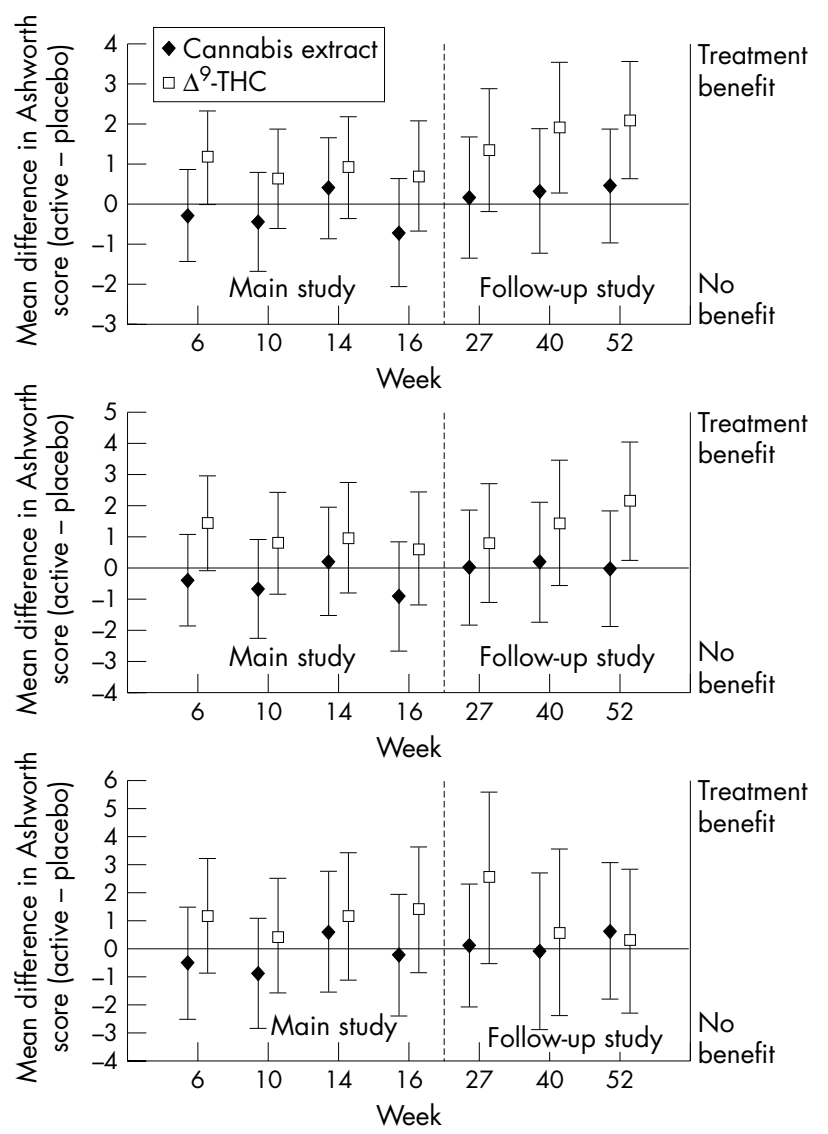

Figure $295 \%$ Confidence intervals for differences in means (active minus placebo) of changes in total Ashworth score at each visit. Positive values favour treatment. In total, 55 patients ( 19 on cannabis extract, 14 $\triangle 9-T H C$, and 22 placebo) started the trial towards the end of the recruitment phase for the main study and were therefore necessarily unable to complete 52 weeks ( 11 visits) of follow up. For these patients, their last Ashworth score (visit 10) was carried forward as their final measured response. Top panel, all patients; Middle panel, elected to continue medication; Bottom panel, elected to discontinue medication.

and was compared using an analysis of variance model, ${ }^{7}$ with treatments as fixed effects. Centres and ambulatory status were also added to the model.

Times taken to complete a 10 metre walk were analysed using the Kruskal-Wallis test, and non-parametric methods were used to produce confidence intervals for the median. The RMI, UKNDS, postal BI, and GHQ-30 were each analysed using non-parametric analysis of variance to compare the groups. EDSS scores were analysed using Fisher's exact test. Category rating scales were analysed using contingency table analysis. Comparison was made between patients choosing to continue on medication and those who chose to discontinue, by baseline Ashworth scores, RMI, EDSS, age, weight, ambulatory status, type of disease, sex, and use of urinary catheter. No adjustments were made for multiple comparisons.

\section{RESULTS}

Ashworth score data were obtained for 502 (80\%) of the 630 patients in the intention to treat sample of the main study: 154 patients on $\Delta^{9}$-THC ( 117 continued medication), 172 on cannabis extract (127 continued), 176 on placebo (111 continued) (fig 1). Mean (SD) difference in Ashworth scores from baseline to end of study were 1.82 (8.12), 0.10 (7.25), and $-0.23(7.87)$ in the $\Delta^{9}$-THC, cannabis extract, and placebo groups respectively. There was no evidence from
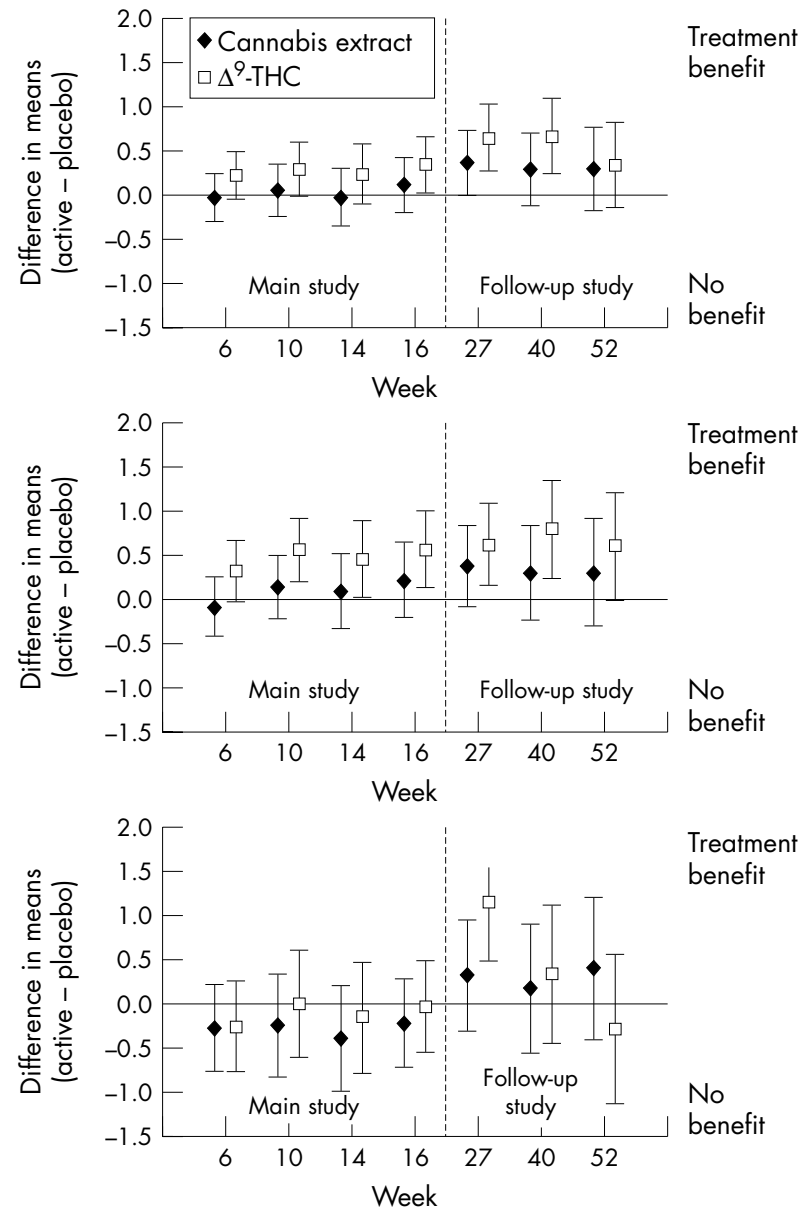

Figure 3 Treatment effect on change in RMI adjusted for baseline, ambulatory status, and treatment centre. Top panel, all patients followed up; middle panel, patients followed up who chose to continue treatment; bottom panel, patients followed up who chose not to continue treatment. Positive values favour treatment. CM, chose to continue medication; DM, chose to discontinue medication. Data are number of episodes (number of patients).

baseline values that patients for whom data were missing at final assessment or those who chose not to continue medication differed from those in whom monitoring and medication continued throughout.

Comparison of the three groups using analysis of variance on the change in total Ashworth score showed evidence of a small treatment effect $(p=0.01$ adjusted for ambulatory status and centre, $\mathrm{p}=0.04$ without adjustment) (fig 2).

Both centre $(p<0.0001)$ and ambulatory status $(p=0.006)$ had an effect on change in Ashworth score, as might be expected due to different sizes of centres and the nature of the ambulatory status, but there was no evidence of an interaction between either treatment and centre, or treatment and ambulatory status. This illustrates that the treatment effect is similar in all centres and in the two ambulatory status groups.

Suggestion of treatment effect was found in the RMI (fig 3), particularly in the $\Delta^{9}$-THC group. This effect was strongest at the week 40 visit. No treatment effects were evident in the BI or UKNDS (all assessed using nonparametric analysis of variance to compare groups). Evidence would suggest that the RMI is the most responsive of the outcome measures used. ${ }^{8}$ In patients who chose to discontinue medication, there were no significant treatment effects in any of the outcome measures. 
Table 1 Category rating scales analysed using contingency table analysis

\begin{tabular}{|c|c|c|c|c|c|c|c|c|c|c|}
\hline & \multicolumn{3}{|c|}{ Improvement } & \multicolumn{3}{|l|}{ Same } & \multicolumn{3}{|c|}{ Deterioration } & \multirow[b]{2}{*}{$\mathbf{p}^{*}$} \\
\hline & $\Delta^{9}-\mathrm{THC}$ & CE & Placebo & $\Delta^{9}-\mathrm{THC}$ & CE & Placebo & $\Delta^{9}-\mathrm{THC}$ & CE & Placebo & \\
\hline Pain $(n=356)$ & $30(28)$ & $38(31)$ & $17(14)$ & $61(56)$ & $53(44)$ & $70(56)$ & $18(16)$ & $31(25)$ & $38(30)$ & 0.002 \\
\hline Shaking $(n=328)$ & $25(26)$ & $38(33)$ & $23(20)$ & $53(55)$ & $56(49)$ & $64(55)$ & 19 (19) & 20 (18) & $30(25)$ & 0.015 \\
\hline Spasms $(n=438)$ & $39(29)$ & $55(36)$ & $35(23)$ & $62(46)$ & 72 (47) & $69(45)$ & $33(25)$ & 25 (17) & 48 (32) & 0.002 \\
\hline Spasticity ( $n=384$ ) & $47(33)$ & $45(29)$ & 27 (17) & $48(34)$ & 66 (42) & $71(46)$ & $47(33)$ & 45 (29) & $58(37)$ & 0.004 \\
\hline Sleep $(n=397)$ & $40(34)$ & $54(38)$ & $36(26)$ & $63(54)$ & $68(48)$ & $80(58)$ & $14(12)$ & $19(14)$ & $23(16)$ & 0.016 \\
\hline Energy $(n=476)$ & $41(24)$ & $36(23)$ & $36(13)$ & $79(56)$ & $87(56)$ & $90(58)$ & $28(20)$ & $33(21)$ & $46(29)$ & 0.004 \\
\hline Tiredness ( $n=422$ ) & $32(25)$ & $26(18)$ & $17(11)$ & 70 (54) & $81(56)$ & $88(60)$ & $28(21)$ & $37(26)$ & $43(29)$ & 0.025 \\
\hline Depression $(n=324)$ & $27(26)$ & $38(34)$ & $20(18)$ & 59 (57) & $55(50)$ & $71(65)$ & 17 (17) & $18(16)$ & 19 (17) & 0.135 \\
\hline Irritability $(n=311)$ & $31(30)$ & $32(30)$ & $20(20)$ & $56(55)$ & $64(60)$ & $61(60)$ & 15 (15) & $11(10)$ & 21 (20) & 0.125 \\
\hline
\end{tabular}

Longer term trends were also seen in the walking time data, with a reduction in walking time, followed by an increase, although statistical significance was not reached. The proportions of patients unable to walk at the end of the study, but who could walk at baseline were 6\%, 14\%, and 13\% in the $\Delta^{9}$-THC, cannabis extract, and placebo groups respectively. Rating scales showed highly significant effects on pain, spasms, spasticity, and sleep, as in the main study (table 1).

Evidence of treatment effects on ratings of shakiness, energy level, and tiredness was found at 27 and 52 weeks, but not on depression or irritability, suggesting wider symptomatic benefit with time, but interpretation is difficult due to unmasking identified in the main study period. There were no major differences between the active treatment groups in these subjective measures, in contrast to the Ashworth and RMI results.

During this study, all hospital admissions were classed as serious adverse events. Most of the 74 serious adverse events reported ( $24 \Delta^{9}$-THC, 27 cannabis extract, 23 placebo; table 2 ) were as expected in this patient population.

Unblinding of treatment was not required for any event. The first phase of the study showed a reduction in relapse rate in the active treatment groups compared with placebo, as measured by the number of relapses requiring hospital admission. This was not sustained in the follow up period. When relapses were identified from all sources (corticosteroid administration, adverse events and serious adverse events), there appeared to be a similar number of relapses in each of the three groups. This also suggests that the reduction in disability seen in the $\Delta^{9}$-THC group cannot be accounted for by a reduction in relapse rate.

There were six deaths during the follow up phase (three from pneumonia, one from carcinoma of the cervix diagnosed after study randomisation, and one each from seizures and ischaemic heart disease). A further patient $\left(\Delta^{9}\right.$-THC group) died in week 42 from urinary/respiratory infections contracted during week 12 of the main study. Overall, there were no major safety concerns. Minor adverse events were reported by 361 patients ( 109 on $\Delta^{9}$-THC, 125 on cannabis extract, 127 on placebo; table 3 ).

Urinalysis for cannabinoids throughout the study demonstrated low levels of illicit cannabis use in the placebo group, with no more than four patients demonstrating urinary cannabinoid presence at any time.

\section{DISCUSSION}

Although patients felt that both cannabis extract and $\Delta^{9}$-THC helped their spasticity (according to rating scales), this was confirmed objectively by the Ashworth score only in the $\Delta^{9}$ THC group. An improvement of approximately 2 points on the Ashworth scale from a baseline mean of around 22 occurred in this group, compared with a placebo deterioration of around 0.2. We consider this change to be small, although the clinical significance of this change from the patient perspective requires further investigation. The reasons for the differences between the two active treatment groups comparing subjective and objective measurements cannot be

Table 2 Frequency of serious adverse events

\begin{tabular}{|c|c|c|c|c|c|c|}
\hline \multirow[b]{2}{*}{ Adverse event } & \multicolumn{2}{|c|}{$\Delta^{9}-\mathrm{THC}(\mathrm{n}=24)$} & \multicolumn{2}{|c|}{ Cannabis extract ( $n=27$ ) } & \multicolumn{2}{|c|}{ Placebo $(n=23)$} \\
\hline & $\mathrm{CM}$ & DM & $\mathrm{CM}$ & DM & $\mathrm{CM}$ & DM \\
\hline Relapse/possible relapse & $8 \dagger$ & 2 & 8 & 1 & 2 & 2 \\
\hline Urinary tract infection & 3 & 0 & 3 & 0 & $3 \ddagger$ & 1 \\
\hline Pneumonia/chest infection & 1 & 0 & $5 \S(1$ died $)$ & 1 (died) & 1 & 1 (died) \\
\hline Seizure & 0 & 0 & 1 (died) & 0 & 1 & 2 \\
\hline Insertion of baclofen pump & 0 & 0 & 1 & 1 & 0 & 0 \\
\hline Limb fracture & 0 & 0 & 1 & 0 & 1 & 0 \\
\hline Other & 8 & 2 & 3 & 2 & 8 & 1 \\
\hline No. of events (no. of patients) & 20 (18) & $4(4)$ & $22(21)$ & $5(5)$ & $16(15)$ & $7(7)$ \\
\hline
\end{tabular}

Data are number of patients unless otherwise indicated. CM, chose to continue medication; DM, chose to discontinue medication. †6 patients; $¥ 2$ patients; $\$ 4$ patients. OOther events were as follows (* indicates where patient chose not to continue trial medication at the end of the main study). Cannabis extract group: blocked/ insertion of suprapubic catheter*; haematuria*; relapse/urinary tract infection; symptoms of nervous breakdown (inappropriate serious adverse event report); viral gastroenteritis. $\Delta^{9}$-THC group: carcinoma of cervix (died)*; cellulitis; dizziness + chest pain (non-cardiac); drug induced cholestasis*; groin abscess; lymph node biopsy (carcinoma of bronchus; died 9 months post-trial); pleural effusion; possible transient ischaemic attack; urinary tract infection and bowel problem; urinary tract infection and relapse. Placebo group: cerebrovascular episode; chest pain; disease progression (not relapse); hospitalisation for physiotherapy; ischaemic heart disease (died); overdose of analgesics; pulmonary embolism*; relapse/cellulitis; review/respite. 
Table 3 Frequency of minor adverse events

\begin{tabular}{|c|c|c|c|c|c|c|}
\hline \multirow[b]{2}{*}{ Adverse event } & \multicolumn{2}{|l|}{$\Delta^{9}$-THC } & \multicolumn{2}{|c|}{ Cannabis extract } & \multicolumn{2}{|l|}{ Placebo } \\
\hline & $\begin{array}{l}C M \\
(n=125)\end{array}$ & $\begin{array}{l}\text { DM } \\
(n=91)\end{array}$ & $\begin{array}{l}C M \\
(n=138)\end{array}$ & $\begin{array}{l}\text { DM } \\
(n=81)\end{array}$ & $\begin{array}{l}C M \\
(n=120)\end{array}$ & $\begin{array}{l}\text { DM } \\
(n=102)\end{array}$ \\
\hline Bladder & $26(16)$ & $9(6)$ & $37(24)$ & $3(3)$ & $42(22)$ & $12(11)$ \\
\hline Depression or anxiety & $11(9)$ & $3(3)$ & $11(11)$ & $3(3)$ & $10(10)$ & $2(2)$ \\
\hline Dizziness or lightheadedness & 19 (17) & $1(1)$ & $27(20)$ & $1(1)$ & $5(4)$ & $1(1)$ \\
\hline Dry mouth & $3(3)$ & $1(1)$ & $2(2)$ & $0(0)$ & $1(1)$ & $1(1)$ \\
\hline Falls & $10(7)$ & $1(1)$ & $10(10)$ & $5(5)$ & $4(3)$ & $4(4)$ \\
\hline Fatigue or sleep disturbance & $15(14)$ & $3(3)$ & $15(15)$ & $3(3)$ & $14(13)$ & $10(8)$ \\
\hline Gastrointestinal tract & $22(17)$ & $3(3)$ & $30(24)$ & $2(2)$ & $14(11)$ & $5(5)$ \\
\hline Infection & $17(14)$ & $7(5)$ & $26(19)$ & $7(5)$ & $20(17)$ & $10(7)$ \\
\hline Memory or concentration & $5(5)$ & $0(0)$ & $5(4)$ & $0(0)$ & $1(1)$ & $1(1)$ \\
\hline Miscellaneous & $17(13)$ & $3(3)$ & $15(11)$ & $9(5)$ & $13(11)$ & $6(5)$ \\
\hline MS relapse or exacerbation & $11(9)$ & $2(2)$ & $13(10)$ & $4(4)$ & $9(9)$ & $5(5)$ \\
\hline Numbness or paraesthesia & $9(8)$ & $2(2)$ & $5(4)$ & $5(5)$ & $5(5)$ & $3(3)$ \\
\hline Other skin problem & $3(3)$ & $0(0)$ & $7(7)$ & $5(4)$ & $10(8)$ & $6(5)$ \\
\hline Pain & $24(18)$ & $5(3)$ & $36(26)$ & $15(11)$ & 20 (14) & $9(9)$ \\
\hline Pressure sores & $1(1)$ & $0(0)$ & $2(2)$ & $1(1)$ & $5(5)$ & $1(1)$ \\
\hline Spasms or stiffness & $30(26)$ & $6(5)$ & $35(23)$ & $10(9)$ & $36(23)$ & $7(7)$ \\
\hline Tremor or lack of coordination & $9(9)$ & $1(1)$ & $4(4)$ & $1(1)$ & $2(2)$ & $3(3)$ \\
\hline Vision symptoms & $4(4)$ & $1(1)$ & $1(1)$ & $4(4)$ & $1(1)$ & $0(0)$ \\
\hline Weakness or reduced mobility & $16(15)$ & $9(7)$ & 20 (19) & $11(7)$ & $24(22)$ & $15(13)$ \\
\hline Total & 252 (87) & 57 (22) & 301 (95) & 89 (30) & $236(85)$ & 100 (42) \\
\hline
\end{tabular}

explained by differences in side effects, which were similar for both active groups. Although the study was not designed to detect differences between the two active treatment groups, it is possible that these differences may be mediated at the receptor level, thus further work is needed to differentiate pharmacodynamic from pharmacokinetic effects.

Recent work suggests that spasticity is a very complex phenomenon, with contributions from patient symptoms, physical functioning, and psychological impact. ${ }^{9}$ The way in which patients' symptoms impact on physical disability is not well understood, and the considerable symptomatic effects seen over the course of this study may have influenced the results seen in disability scores. Whether any effect on disability could be explained solely by a reduction in spasticity requires further long term studies. The complex interaction between symptoms and disability has affected previous studies, notably the DATATOP study of selegiline in the treatment of Parkinson's disease. ${ }^{10}$ Although experimental evidence demonstrates a potential neuroprotective effect of cannabinoids, ${ }^{5}$ more work is needed to define neuroprotection before such a role can be tested in humans.

This study was designed primarily as a short term symptomatic study, with a voluntary continuation phase leading to incomplete long term follow up data. Two important sources of bias that require consideration in the interpretation of the long term follow up data are the losses to follow up (around $20 \%$, with some differential loss in the $\Delta^{9}$-THC group), and the discontinuation of medication after the first phase in a proportion of patients $\left(35 \% \Delta^{9}-\right.$ THC, $33 \%$ cannabis extract, $41 \%$ placebo). Side effects were similar in both cannabis extract and $\Delta^{9}$-THC groups, and there is nothing to suggest that there were any differences in reasons for loss to follow up between the two active treatment arms.

In order to limit any self selection bias, data were analysed by treatment group irrespective of whether patients continued treatment. The absence of difference in characteristics between the group continuing medication and those originally randomised to receive treatment also suggests that patients continuing medication did not have a substantially different disease course. The numbers deciding to stop trial medication were similar in each of the three study arms, but the reasons given were different. Of the patients in the placebo group who decided not to continue treatment, 74\% felt that the medication produced no benefit, compared with $45 \%$ in the cannabis extract and $42 \%$ in the $\Delta^{9}$-THC groups. Adverse effects were more commonly cited in the active treatment arms. We evaluated degree of masking in the main study, which demonstrated that, although patients and treating physicians correctly guessed treatment allocation in the active treatment arms more often than in the placebo group, the assessors remained blinded to treatment allocation. We did not evaluate further potential unmasking over the 12 month follow up period as we felt it unlikely to alter after the first 15 week period. As in the main study, all results need to be interpreted with a degree of caution owing to potential bias from unblinding. However, the Ashworth score and RMI were both performed by assessors who were likely to have remained blinded over the course of the study.

There is now an urgent need to construct a long term study in progressive MS to establish whether $\Delta^{9}$-THC has a role in long term disease management or may be restricted to symptomatic amelioration in this disabling condition.

\section{ACKNOWLEDGEMENTS}

We would like to thank all patients who took part in the study. We acknowledge the assistance of the MRC Clinical Trials Unit in the management and design of the study, the CAMS management team in Plymouth (especially J Willcocks, S Varley, C Brooke, M Warner, N Wheeler, and F Coates) and Dr Jeremy Hobart for his comments on the manuscript. Grateful thanks also to Mr M Marner and Dr M Priston (Pharmacy Department, Derriford Hospital) and to all members of the trial steering committee and the data monitoring and ethics committee. Finally, our thanks go to Solvay Healthcare Ltd and the Institute for Clinical Research, IKF, Berlin.

\section{Authors' affiliations}

H P Sanders, D E Wright, Department of Mathematics and Statistics, University of Plymouth, Plymouth, Devon, UK

J P Zajicek, P J Vickery, W M Ingram, S M Reilly, A J Nunn, L J Teare, P J Fox, Peninsula Medical School, Plymouth, Devon, UK

A J Thompson, National Hospital for Neurology and Neurosurgery, University College Hospitals, London, UK

Competing interests: Professor Zajicek received support from Solvay Healthcare Ltd and the Institute for Clinical Research, IKF, Berlin to attend meetings with the UK MHRA regarding licensing. 
The following contributed patients to the CAMS study as part of the UK MS Research Group: Dr S A Ahmed, Dr E J W McClemont (Community Rehabilitation Centre, Lincoln); Dr D Barnes, Dr N Stoy, Dr D Wren (Atkinson Morley's Hospital, London); Professor D Bates (Royal Victoria Infirmary, Newcastle); Dr M Boggild (Walton Centre, Liverpool); Dr C Constantinescu (University Hospital, Nottingham); Dr E Fathers (Taunton and Somerset Hospital); Dr H Ford, Dr M Johnson (St James' University Hospital, Leeds); Dr D A Francis, Dr J B Winer (Queen Elizabeth Hospital, Birmingham); Dr C P Hawkins (North Staffordshire Royal Infirmary); Dr S Hawkins, Dr A G Droogan (Royal Victoria Hospital, Belfast); $\operatorname{Dr} S$ J L Howell, Dr S Price (Royal Hallamshire Hospital, Sheffield); Dr D Kidd (Hertford County Hospital); Dr L A Loizou (Pinderfields General Hospital, Wakefield); Dr P G Mattison (Ayrshire Central Hospital; Dr B McLean, Dr J Morgan (Royal Cornwall Hospital, Truro); Dr J O'Riordan, Dr R Swingler, Dr K White (Ninewells Hospital and Medical School, Dundee); Dr J Palace (Raddliffe Infirmary, Oxford); Dr G D Perkin (Charing Cross Hospital, London); Dr I F Pye, Dr B R Kendall (Leicester Royal Infirmary); Dr C Rickards (Morriston Hospital, Swansea); Dr N Robertson, Dr TAT Hughes (University Hospital of Wales and Rookwood Hospital, Cardiff); Professor N J Scolding, Dr J Burrow (Frenchay Hospital, Bristol); Dr M Sharief, Dr O Seidi (Guy's Hospital, London); Dr A Shehu (Coventry and Warwickshire Hospital, Coventry); Dr E Silber (Queen Elizabeth Hospital, Woolwich); Dr P R Talbot (Hope Hospital, Manchester); Professor A J Thompson (Institute of Neurology, Queen Square, London); $\mathrm{Dr} J$ Thorpe, $\mathrm{Dr} I$ Bjornson (Addenbrooke's Hospital, Cambridge); Dr P Tidswell, Dr I Redmond (Royal Preston Hospital); Dr S A Wasti (Community Rehabilitation and Respite Unit, Barnsley); Dr S J Wroe, Dr K Powell (Ipswich Hospital); Professor J P Zajicek (Derriford Hospital, Plymouth).
Steering Committee: D Chadwick (chair), D Jones, T Meade, T Moffat, A Nunn, M O'Donovan, A Thompson, J Zajicek. Data Monitoring and Ethics Committee: C. Polman (chair), P. Stroner, C. Warlow.

\section{REFERENCES}

1 Consroe P, Musty R, Rein J, et al. The perceived effects of smoked cannabis on patients with multiple sclerosis. Eur Neurol 1977;38:44-8.

2 Skaper SD, Buriani A, Dal Toso R, et al. The ALIAmide palmitoylethanolamide and cannabinoids, but not anandamide, are protective in a delayed postglutamate paradigm of excitotoxic death in cerebellar granule neurons. Proc Natl Acad Sci USA 1996;93:3984-9.

3 Hampson AJ, Grimaldi M, Axelrod J, et al. Cannabidiol and (-)delta9tetrahydrocannabinol are neuroprotective antioxidants. Proc Natl Acad Sci SA 1998;95:8268-73.

4 Panikashvili D, Simeonidou C, Ben-Shabat S, et al. An endogenous cannabinoid (2-AG) is neuroprotective after brain injury. Nature 2001; 413:527-31.

5 Pryce G, Ahmed Z, Hankey DJ, et al. Cannabinoids inhibit neurodegeneration in models of multiple sclerosis. Brain 2003;126:2191-202.

6 Zajicek J, Fox P, Sanders H, et al. Cannabinoids for treatment of spasticity and other symptoms related to multiple sclerosis (CAMS study): multicentre randomised placebo-controlled trial. Lancet 2003;362:1517-26.

7 Gardner MJ, Altman DG. Statistics with confidence. London: British Medical Journal, 1989.

8 Wiles CM, Newcombe RG, Fuller KJ, et al. Controlled randomised crossover trial of the effects of physiotherapy on mobility in chronic multiple sclerosis. J Neurol Neurosurg Psychiatry 2001;70:174-9.

9 Riazi A, Fox P, Vickery J, et al. Developing a patient-based measure of the impact of spasticity in multiple sclerosis. Multiple Sclerosis 2003;9:S151.

10 Stocchi F, Olanow CW. Neuroprotection in Parkinson's disease: clinical trials. Ann Neurol. 2003;53: S87-97; discussion S97-89, (suppl 3). 